\title{
Remote electron plasmon polaron in graphene
}

\author{
P. M. Krstajići ${ }^{1,2}$ and F. M. Peeters ${ }^{2}$ \\ ${ }^{1}$ Institute of Microelectronic Technologies and Single Crystals (IHTM), Belgrade University, Njegoševa 12, 11000 Belgrade, Serbia \\ ${ }^{2}$ Departement Fysica, Universiteit Antwerpen, Groenenborgerlaan 171, B-2020 Antwerpen, Belgium
}

(Received 18 November 2011; published 27 February 2012)

\begin{abstract}
The Coulomb interaction and the correlation of a remote electron with a single layer of graphene is investigated in the presence of a magnetic field applied perpendicular to the graphene layer. The remote electron polarizes the electron gas in the graphene layer, which we describe in terms of excitations of virtual plasmons in graphene. The composite quasiparticle formed by electron plus polarization is called a plasmon polaron. The ground-state energy of this quasiparticle is calculated within perturbation theory for remote electrons in different environments.

DOI: 10.1103/PhysRevB.85.085436

PACS number(s): 73.23.-b, 71.38.-k, 73.25.+i, 72.80.Vp
\end{abstract}

\section{INTRODUCTION}

There has been growing interest in the electronic properties of a single layer of graphene, especially those related to the properties of plasmons, ${ }^{1}$ dynamical screening effects, ${ }^{2}$ and plasmon-phonon coupling. ${ }^{3}$ Recently, the interaction between electrons and plasmons was also investigated both theoretically ${ }^{4}$ and experimentally, ${ }^{5}$ and the concept of a quasiparticle termed plasmaron was introduced as a consequence of this interaction. It is well known that the low-energy dispersion in graphene is linear in the electron momentum, in stark contrast to both two-dimensional (2D) and three-dimensional (3D) conventional semiconductors. This comes as a consequence of the chiral and massless (Dirac-like) properties of charge carriers in this material. Thus, not only the lower dimensionality of this material but also the band structure give rise to many unusual phenomena such as the anomalous quantum Hall effect, ${ }^{6,7}$ Klein tunneling, ${ }^{8,9}$ and Zitterbewegung. ${ }^{10,11}$ Graphene also possess a large electron mobility at room temperature which motivates further research for possible applications. The important quantities related to electronic properties such as, for instance, the dielectric function and phonon and plasmon dispersions must be carefully derived since they often have no analog in usual 2D or 3D semiconductors.

In this paper we extend the previous study ${ }^{12}$ of the interaction of a remote electron with a $2 \mathrm{D}$ electron gas (2DEG) to the case in which the 2DEG is replaced by a single layer of graphene. Though the essential elements of physics are similar, the electronic properties of graphene are significantly different. It is assumed that the remote electron is placed at a small distance $d$ away from the graphene layer and that it will induce a polarization in graphene. The polarization is expressed in terms of excitation of virtual plasmons. The interaction of the electron with graphene results in the creation of a composite quasiparticle termed plasmon-polaron. Essentially it is the binding of an external electron to the graphene surface. This quantity can be measured in a manner similar to the binding energy of surface polarons, in which an electron outside the crystal is bound to the surface of the crystal. ${ }^{13}$ The energy spectrum is obtained by shooting electrons parallel to the graphene layer. All these facts give an impetus for further investigation on tunneling into (multilayer) graphene under perpendicular magnetic fields.

We organize the paper as follows. In Sec. II we present the theoretical model and derive relevant expressions for the interaction and the coupling between the remote electron and the graphene layer. In the subsequent section, Sec. III, the numerical calculations of the screening wave vector, Fermi energy, and the energy difference due to a perpendicular magnetic field are presented for concrete systems. Finally, we summarize our conclusions in Sec. IV.

\section{THEORETICAL MODEL}

Let us consider a remote electron that is placed at a distance $d$ away from graphene. The presence of the electron induces a modulation of the electron density $n(\mathbf{r})$ in graphene. The electron density in graphene is given by

$$
n(\mathbf{r})=\sum_{j=1}^{N} \delta\left(\mathbf{r}-\mathbf{r}_{j}\right)=\frac{1}{\Omega} \sum_{\mathbf{k}} n_{\mathbf{k}} \exp (i \mathbf{k} \cdot \mathbf{r}),
$$

with $\mathbf{r}=(x, y)$ being the $2 \mathrm{D}$ position vector, $N$ being the total number of electrons, and $\Omega$ being the system area. Furthermore, $n_{\mathbf{k}}$ is the Fourier coefficient with 2D wave number $\mathbf{k}$. The fluctuations of the density are described in terms of collective excitations of the system, which in our case are magnetoplasmons. When these excitations are formulated in the quantum mechanical sense, the Fourier coefficient $n_{\mathbf{k}}$ is expressed via annihilation $\left(a_{\mathbf{k}}\right)$ and creation operators $\left(a_{\mathbf{k}}^{\dagger}\right)$ for each magnetoplasmon as follows: $n_{\mathbf{k}}=\lambda_{\mathbf{k}}\left(a_{\mathbf{k}}+a_{-\mathbf{k}}^{\dagger}\right)$, where the coefficient $\lambda_{\mathbf{k}}$ will be defined later. The Hamiltonian of the remote electron in a magnetic field that interacts with such collective excitations in the graphene layer is given by

$$
H=\frac{1}{2 m_{e}}\left(\mathbf{p}+\frac{e}{c} \mathbf{A}(\mathbf{r})\right)^{2}+\sum_{\mathbf{k}} \hbar \omega_{k} a_{\mathbf{k}}^{\dagger} a_{\mathbf{k}}+H_{\mathrm{int}} .
$$

The first term is the Hamiltonian of the remote electron in a magnetic field, while the second term describes the magnetoplasmons. The mass of the remote electron is, in general, not the free electron mass $m_{0}$ but $m_{e}=m_{\mathrm{eff}} m_{0}$, where $m_{\text {eff }}$ is the dimensionless effective mass. Further, the interaction Hamiltonian $H_{\text {int }}$ can be determined by evaluating the potential $U_{i n}(\mathbf{r}, z)$ induced by the remote electron and is given by the Poisson's equation

$$
\left(\nabla_{2 D}^{2}+\partial_{z}^{2}\right) U_{i n}(\mathbf{r}, z)=-\frac{4 \pi e}{\kappa}\left\{n(\mathbf{r})-n_{s}\right\} \delta(z),
$$


where $\nabla_{2 D}^{2}$ is the 2D Laplacian, $\kappa$ is the dielectric constant of graphene, and $n_{s}=N / \Omega$ is the average electron density of the graphene layer. The solution is given by

$$
U_{i n}(\mathbf{r}, z)=-\frac{1}{\Omega} \sum_{\mathbf{k}} \frac{2 \pi e}{\kappa k} \exp (-k|z|) n_{\mathbf{k}} \exp (i \mathbf{k} \cdot \mathbf{r}) .
$$

The interaction Hamiltonian with the remote electron at $z=d, H_{\text {int }}=-e U_{i n}(\mathbf{r}, z=d)$, takes the following form

$$
H_{\text {int }}=\sum_{\mathbf{k}} \frac{V_{\mathbf{k}}}{\sqrt{\Omega}} \exp (i \mathbf{k} \cdot \mathbf{r})\left(a_{\mathbf{k}}+a_{-\mathbf{k}}^{\dagger}\right),
$$

where we have introduced the interaction strength $V_{\mathbf{k}}$ given by

$$
V_{\mathbf{k}}=\frac{2 \pi e^{2}}{\sqrt{\Omega} \kappa k} \lambda_{\mathbf{k}} \exp (-k d) .
$$

On the other hand, the Hamiltonian describing the motion of electrons in the graphene single layer is given by

$$
H_{g r}=\sum_{j=1}^{N} v_{F} \boldsymbol{\sigma} \cdot\left(\hbar \mathbf{k}_{j}-e \mathbf{A}\left(\mathbf{r}_{j}\right) / c\right)+\sum_{j \neq i}^{N} \frac{e^{2}}{2 \kappa\left|\mathbf{r}_{i}-\mathbf{r}_{j}\right|},
$$

where $\sigma=\left(\sigma_{x}, \sigma_{x}\right)$ are the $2 \mathrm{D}$ Pauli matrices. In order to determine the coefficient $\lambda_{\mathbf{k}}$ and the magnetoplasmon frequency we will use the $f$-sum rule derived for graphene. It is obtained by evaluating the expectation value of the double commutator $\left[\left[H, n_{\mathbf{k}}\right], n_{-\mathbf{k}}\right]$ in two different ways. ${ }^{14}$ First, it is known that the relation $\langle n|C| m\rangle=\left(E_{n}-E_{m}\right)\langle n|A| m\rangle$ holds for any commutator with the Hamiltonian, that is, $C=[A, H]$. Then it can easily be proven that

$$
\left\langle n\left|\left[\left[H, n_{\mathbf{k}}\right], n_{-\mathbf{k}}\right]\right| 0\right\rangle=2 \sum_{n} \hbar \omega_{n 0}\left|\left\langle n\left|n_{\mathbf{k}}\right| 0\right\rangle\right|^{2},
$$

where $\hbar \omega_{n 0}=E_{n}-E_{0}$. On the other hand, from an explicit evaluation ${ }^{15}$ of the double commutator, we find the following relation:

$$
\sum_{n} \hbar \omega_{n 0}\left|\left\langle n\left|n_{\mathbf{k}}\right| 0\right\rangle\right|^{2}=n_{s}^{\prime} \frac{\Omega \Lambda_{E} k^{2}}{4 \pi} .
$$

Here $n_{s}^{\prime}$ is the concentration per unit cell $n_{s}^{\prime}=n_{s} 3 \sqrt{3} a^{2} / 2$, where $a=a_{C-C}$ is the carbon-carbon distance $a=1.42 \AA$. The quantity $\Lambda_{E}$ is the energy cutoff and is of the order of the bandwidth ${ }^{15} \Lambda_{E} \approx W$, where ${ }^{16} W=\sqrt{\sqrt{3} \pi} t$ and $t$ is the hopping energy $t=2.8 \mathrm{eV} .{ }^{10}$ Notice that the factor $\Omega /(2 \pi)$ appears when going from summation to integration over $\mathbf{k}$ (see Ref. 15 for details). Finally, one may argue that there is only one collective excitation for each wave vector $\mathbf{k}$ within the plasmon pole, so that one can put $\omega_{n 0}=\omega_{\mathbf{k}}$, which leads to

$$
\hbar \omega_{\mathbf{k}} \lambda_{\mathbf{k}}^{2}=n_{s}^{\prime} \frac{\Omega \Lambda_{E} k^{2}}{4 \pi} .
$$

The extension to the case in which a magnetic field is present is even more straightforward than the case of conventional semiconductors since the kinetic term is linear in the vector potential $\mathbf{A}(\mathbf{r})$. Hence it will not produce any additional term on either side of Eq. (10). By inserting the expression for $\lambda_{\mathbf{k}}$ into Eq. (6), one arrives at

$$
V_{\mathbf{k}}=\frac{2 \pi e^{2}}{\kappa} \sqrt{\frac{n_{s}^{\prime} g_{v}}{\hbar \omega_{\mathbf{k}}} \frac{W}{8 \pi}} e^{-k d}
$$

The remote electron also induces an electrostatic potential on its own, which obeys Poisson's equation, while its form can be written as

$$
U_{e x}(\mathbf{r}, z)=\frac{1}{\Omega} \sum_{\mathbf{k}} U_{\mathbf{k}}^{e x} \exp (i \mathbf{k} \cdot \mathbf{r}),
$$

where

$$
U_{\mathbf{k}}^{e x}(z)=\frac{2 \pi e^{2}}{k \kappa} \exp (-k|d-z|) .
$$

Within the self-consistent field approximation, the total potential in the graphene layer at $z=0, U_{\mathbf{k}}^{\text {tot }}(0)$ is given as a sum of the external potential $U_{\mathbf{k}}^{e x}(0)$ and the induced potential $U_{\mathbf{k}}^{\text {in }}(0)$, such that $U_{\mathbf{k}}^{\text {tot }}(0)=U_{\mathbf{k}}^{e x}(0) / \epsilon(k)$, where $\epsilon(k)$ is the dielectric function. The induced potential in the region outside the graphene layer is given by

$$
U_{\mathbf{k}}^{i n}(z)=-\exp (-k|z|) \frac{\epsilon(k)-1}{\epsilon(k)} U_{\mathbf{k}}^{e x}(0) .
$$

On the other hand, in classical electrostatics the interaction energy between a test charge placed at the point $(\mathbf{r}, z)=(0, d)$ and the induced polarization is given by $W_{c l}=$ $(-e / 2) U_{i n}(\mathbf{r}=0, z=d)$. Furthermore, if the motion of the test charge at $(\mathbf{r}, z)=(0, d)$ is determined by the Hamiltonian in Eq. (2), second-order perturbation theory gives $W_{q m}=$ $\sum\left|V_{\mathbf{k}}\right|^{2} /\left(\Omega \hbar \omega_{\mathbf{k}}\right)$. Upon comparing the two energies $W_{c l}$ and $W_{q m}$ one finally obtains

$$
\omega_{k}^{2}=\omega_{0}^{2} \frac{\epsilon(k)}{\epsilon(k)-1} .
$$

The first term is the usual plasmon frequency in graphene, $\omega_{0}=\sqrt{\alpha}\left(g_{s} g_{v} \pi n_{s}\right)^{1 / 4} \sqrt{q} v_{F}$, where $g_{s}=2$ and $g_{v}=2$ are the degeneracy factors for spin and valley (pseudospin) degrees of freedom. Note at this point that the plasma frequency in graphene depends on the electron density like $\omega_{0} \propto n^{1 / 4}$, whereas in conventional semiconductors the dependence is $\omega_{0} \propto n^{1 / 2}$. The second term in the previous equation, the factor $\epsilon(k) /(\epsilon(k)-1)$, describes the modulation associated with the excitation of the electron gas at finite $k$. It is believed that the transport properties in graphene supported by a $\mathrm{SiO}_{2}$ substrate are dominated by charged impurity scattering. Then, it can be proven that in the long wavelength limit $(q \rightarrow 0)$ and within the random phase approximation (RPA) the dielectric function can be approximated by ${ }^{2}$

$$
\epsilon(k)=1+\frac{q_{s}}{k},
$$

where $q_{s}$ is the screening wave vector. The dielectric function has the same functional form as in conventional 2D semiconductors, but $q_{s}$ depends in a different manner on the parameters of the material. In the case in which a magnetic field is present, the screening wave vector is given by

$$
q_{s}=q_{s 0} \frac{D(E)}{D_{0}(E)},
$$

where $q_{s 0}=g_{s} g_{v} e^{2} k_{F} /\left(\kappa \hbar v_{F}\right)$ is the Thomas-Fermi wave vector in the absence of a magnetic field and $D(E)$ and $D_{0}(E)$ are the densities of states in the presence and in the absence of a magnetic field. The density of states in graphene for 
$\mathbf{B}=0$ is given by $g_{s} g_{v}|E| /\left(2 \pi\left(\hbar v_{F}\right)^{2}\right)$, while for the case in which a magnetic field and short-range impurity scattering are present ${ }^{17}$ it can be expressed as a series of the Gaussian functions

$$
D(E)=\sum_{n=0}^{\infty} \sum_{s, \sigma} \frac{1}{2 \pi l_{B}^{2}} \frac{1}{\Gamma \sqrt{\pi}} e^{-\left(E-E_{n s \sigma}\right)^{2} / \Gamma^{2}} .
$$

The summation runs over both pseudospin $(s= \pm 1)$ and (usual) spin $(\sigma= \pm 1)$ degrees of freedom. The broadening of the levels, $\Gamma$, is assumed to result from scattering on short-range impurities and is estimated as ${ }^{18} \Gamma=\sqrt{2 / A} \hbar \omega_{c}$, with the dimensionless parameter $A$. The parameter $A$ depends on the concentration of impurities and lies in the range $A=50-100$, whereas it has larger values for cleaner samples. While the cyclotron orbit length is the same as in usual semiconductors, $l_{B}=\sqrt{\hbar c /(e B)}$, the cyclotron frequency in graphene is given as $\omega_{c}=\sqrt{2} v_{F} / l_{B}$. As is well known, the Landau levels in graphene are not evenly spaced but rather given as $E_{n s \sigma}=s \sqrt{n} \hbar \omega_{c}+\sigma \mu_{B} B / 2$, where $\mu_{B}$ is the Bohr magneton. The zeroth level is equally shared between the valence and conduction band so that its degeneracy is twice as small as the valley index $s\left(g_{v 0}=1\right)$. The position of the Fermi level $E_{F}$ is determined by the electron concentration and at zero temperature is given by

$$
n_{s}=\int_{-\infty}^{E_{F}} d E D(E)
$$

This formula is also valid when a magnetic field is present and at larger temperatures since the cyclotron energy is usually significantly larger than the thermal energy excitation $\hbar \omega_{c} \gg$ $k_{B} T$. Having found the critical physical parameters of both graphene and the remote electron, one may readily calculate the ground-state energy of the remote electron interacting with the graphene layer. This interaction is weak, and therefore we are allowed to apply second-order perturbation theory to the Hamiltonian, Eq. (2). The interaction term $H_{\text {int }}$ introduces a shift in the ground-state energy,

$$
E=\frac{1}{2} \hbar \omega_{c e}+\delta E,
$$

with

$$
\delta E=-\frac{1}{\Omega} \sum_{\mathbf{k}}\left|V_{\mathbf{k}}\right|^{2} \sum_{m} \frac{\left|M_{0 m}\right|^{2}}{\hbar \omega_{\mathbf{k}}+m \hbar \omega_{c e}} .
$$

Note that the cyclotron energy of the remote electron (which is outside) is now given as $\omega_{c e}=e B /\left(m_{e} c\right)$. The analytical form of the matrix element is ${ }^{19}\left|M_{0 m}\right|^{2}=u^{m} e^{-u} / m$ !. Since it contains the factor $u^{m} / m$ ! like in the Taylor series of the exponential function $\exp (u)$, the previous relation can be recast in a more appropriate way:

$$
\delta E=-\int_{0}^{\infty} d k \frac{k\left|V_{\mathbf{k}}\right|^{2}}{2 \pi \hbar \omega_{\mathbf{k}}} \int_{0}^{\infty} d \tau \exp \left\{-\tau-k^{2} D_{B}\left(\frac{\tau \omega_{c e}}{\omega_{\mathbf{k}}}\right)\right\} .
$$

where $D_{B}(\xi)=\left(l_{B}^{2} / 2\right)[1-\exp (-\xi)]$.

\section{NUMERICAL RESULTS}

The numerical calculations are carried out for a doped graphene layer and the remote electron placed at the distance

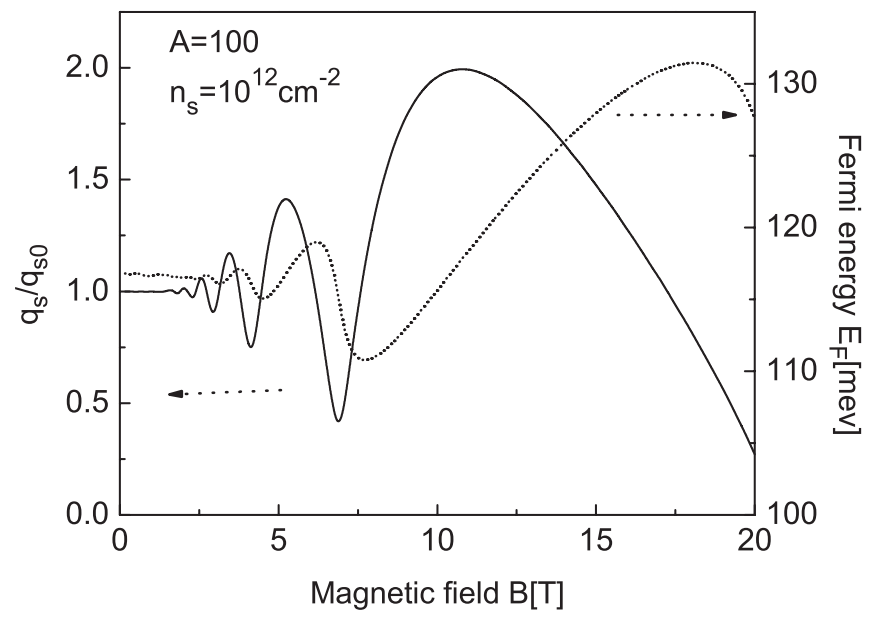

FIG. 1. The dependence of the screening wave vector $q_{s}$ (solid curve) and the Fermi level $E_{F}$ (dotted curve) on the magnetic field $B$ (right axis). The wave vector $q_{s}$ is normalized to the value $q_{s 0}$ for $B=0$. The other parameters are given in the graph.

$d=20 \mathrm{~nm}$ outside the layer. The remote electron is assumed to have a parabolic energy dispersion, with the effective mass equal to that in GaAs, $m_{\mathrm{eff}}=0.067$. Later, other cases will be considered. First, in Fig. 1 we show the screening wave vector $q_{s}$ and the Fermi level (right axis) as a function of the magnetic field, for an electron density $n_{s}=10^{12} \mathrm{~cm}^{-2}$. The position of the Fermi energy varies with the occupation of the Landau levels. Note that in contrast to the case of usual semiconductors, the zeroth Landau level is always placed at the Dirac point $\left(E_{0}=0\right)$, and its degeneracy is twice smaller than that of the other Landau levels. In the limit of a small magnetic field, the screening length reduces to the Thomas-Fermi value $q_{s}=q_{s 0}=6.2 \times 10^{8} \mathrm{~m}^{-1}$ (corresponding length is $\lambda_{T F}=$ $1.6 \mathrm{~nm}$ ). In Fig. 2, we show the correction in the energy of the remote electron $\delta E$ vs magnetic field $B$, for various values

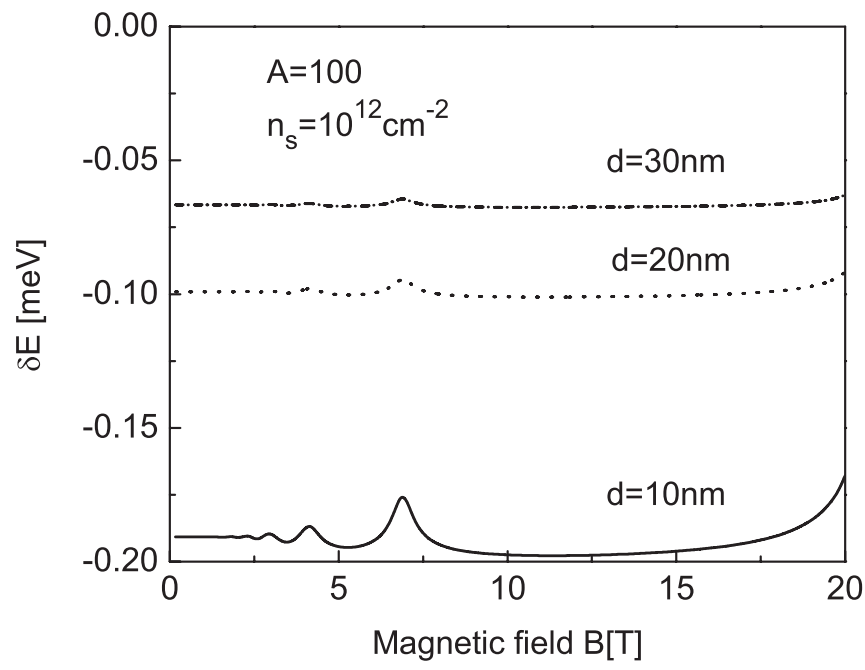

FIG. 2. The correction in the energy of the remote electron $\delta E$ vs magnetic field $B$, for various values of $d: d=10 \mathrm{~nm}$ (solid curve), $d=20 \mathrm{~nm}$ (dotted curve), and $d=30 \mathrm{~nm}$ (dash-dotted curve). The graphene electron density was kept constant $n_{s}=10^{12} \mathrm{~cm}^{-2}$, while the effective mass is $m_{\text {eff }}=0.067$. 


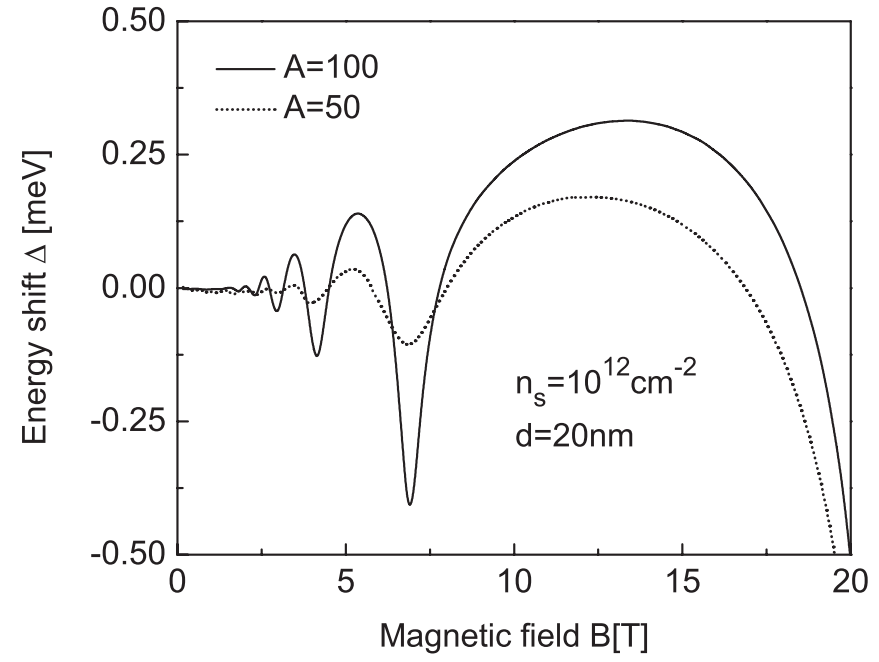

FIG. 3. The remote electron energy shift due to the magnetic field $\Delta=W(d, B)-W(d, B=0)$ vs magnetic field $B$, at a fixed electron concentration $n_{s}=10^{12} \mathrm{~cm}^{-2}$ and a distance $d=20 \mathrm{~nm}$. The solid and dotted curves correspond to two different values of the Landau level broadening parameter, $A=100$ and $A=50$ respectively. The effective mass of the remote electron is $m_{\text {eff }}=0.067$.

of the distance $d: d=10 \mathrm{~nm}$ (solid curve), $d=20 \mathrm{~nm}$ (dotted curve), and $d=30 \mathrm{~nm}$ (dash-dotted curve). The absolute value of the energy shift decreases with the distance as expected, while the peaks follow that of the screening wave vector $q_{s}$.

When an electron tunnels into the graphene layer, the electron gains Coulomb energy $W(d, B)=E(d, B)-E(0, B)$. Since neither the ground-state energy of the electron, $1 / 2 \hbar \omega_{c e}$, nor the spin term depends on $d$, the energy difference $W(d, B)$ can also be expressed as ${ }^{12} W(d, B)=\delta E(d, B)-\delta E(0, B)$. We are further interested in the influence of the magnetic field (i.e., its contribution to the electron energy), so that $\Delta(d, B)=$ $W(d, B)-W(d, 0)$ will be calculated and analyzed. The numerical values of $\Delta$ are given in Fig. 3 as a function of the magnetic field for two different values of the dimensionless parameter $A: A=100$ (solid curve) and $A=50$ (dotted curve). Recall that the value of $A$ depends on the concentration of impurities and is larger for cleaner samples. The shape of the curve is similar to the case in which the graphene layer is replaced by a $2 \mathrm{DEG}$, but note that the positions of the peaks are different. This is due to the different spacing of the Landau levels in graphene following the square root law $E_{n} \propto \sqrt{n}$. The peaks are essentially caused by the variation of the screening wave vector (with $B$ ), since the plasma frequency and hence the interaction coupling $V_{k}$ depend on the factor $\epsilon(k) /(\epsilon(k)-1)$.

Next, we present in Fig. 4 the calculation of the energy shift as a function of the distance $d$, for three values of the magnetic field: $B=5 \mathrm{~T}$ (solid curve), $B=15 \mathrm{~T}$ (dashed curve), and $B=30 \mathrm{~T}$ (dotted curve). The other parameters used in the calculation are, as usual, $n_{s}=10^{12} \mathrm{~cm}^{-2}$ and $A=100$. Notice that for small magnetic fields the value of $\Delta$ saturates for larger distances. This can be explained partly by the fact that the energy difference $\Delta$ is mainly determined by the magnetic field and partly by the fact that the factor $\exp (-k d)$ is present in both terms $W(d, B)$ and $W(d, 0)$. For a large magnetic field, $B=30 \mathrm{~T}$, the difference is negative and saturates also but to a large negative value (as can also been seen from Fig. 3).

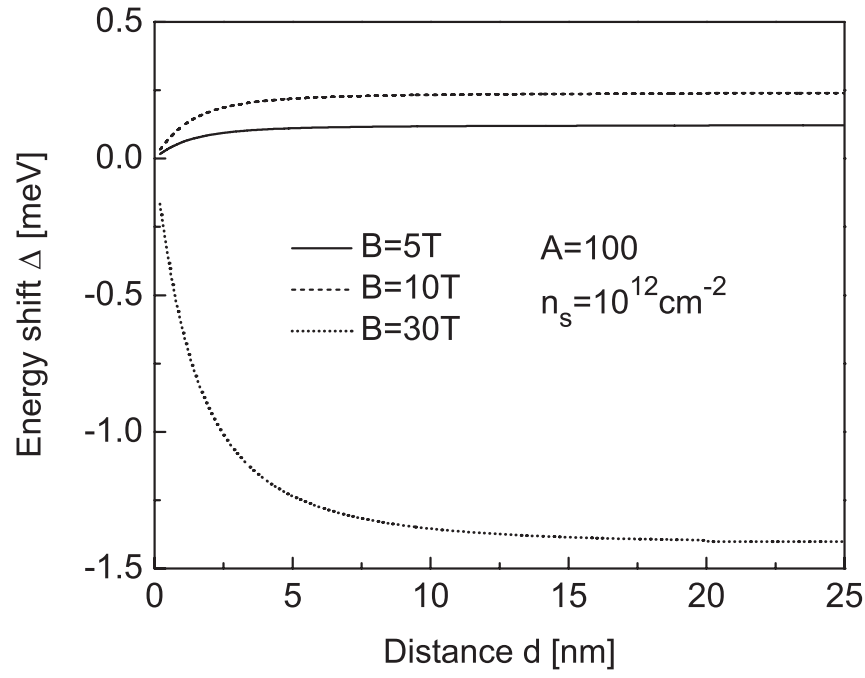

FIG. 4. The energy shift $\Delta$ as a function of the distance $d$ of the remote electron to graphene for three values of the magnetic field: $B=5 \mathrm{~T}$ (solid curve), $B=15 \mathrm{~T}$ (dashed curve), and $B=30 \mathrm{~T}$ (dotted curve). Parameters are $n_{s}=10^{12} \mathrm{~cm}^{-2}$ and $A=100$.

In addition, the values of the energy shift could be useful when the distance $d$ is an integer multiple of the interlayer distance in graphite $d_{0}=3.1 \AA$, and the remote electron now has a Dirac-like energy dispersion. This is important when we consider, for instance, a bilayer or a multilayer graphene system. Figure 5 shows such results for $d=0 d_{0}$ (solid curve), $d=1 d_{0}$ (dashed curve), $d=2 d_{0}$ (dotted curve), and $d=3 d_{0}$ (dash-dotted curve). As usual, the electron density was kept constant at the value $n_{s}=10^{12} \mathrm{~cm}^{-2}$, whereas the dimensionless parameter $A$ has the value of 100 . The peaks are more pronounced since the values of $d$ are small compared to the previous case.

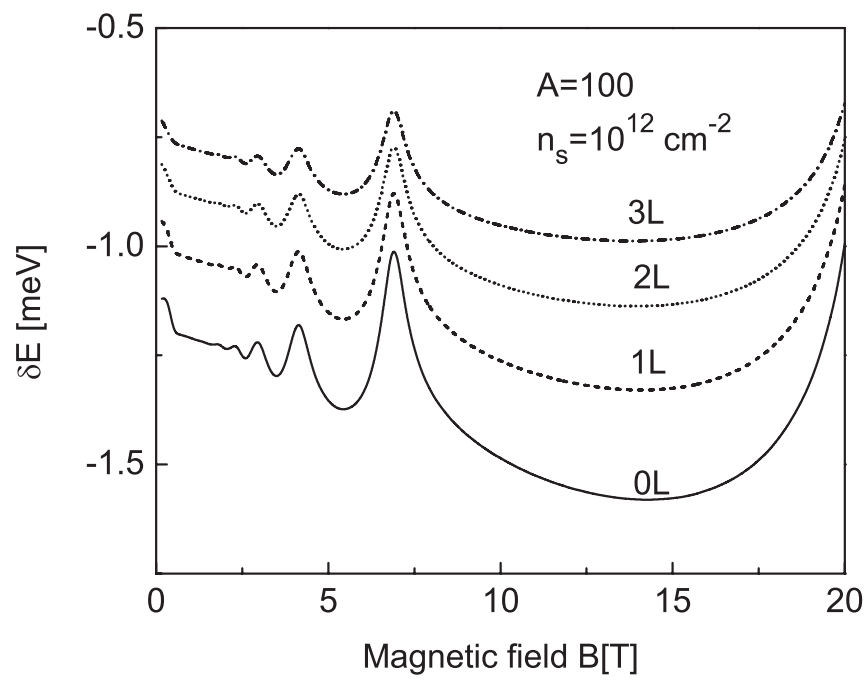

FIG. 5. The correction in the energy $\delta E$ vs magnetic field $B$, for different values of $d$ as integer multiple of interlayer distance $d_{0}=3.1 \AA$, of a multilayer graphene: $d=0 d_{0}$ (solid curve), $d=1 d_{0}$ (dashed curve), $d=2 d_{0}$ (dotted curve), and $d=3 d_{0}$ (dash-dotted curve). The graphene electron density was kept constant $n_{s}=$ $10^{12} \mathrm{~cm}^{-2}$, while the parameter $A$ has the value $A=100$. 


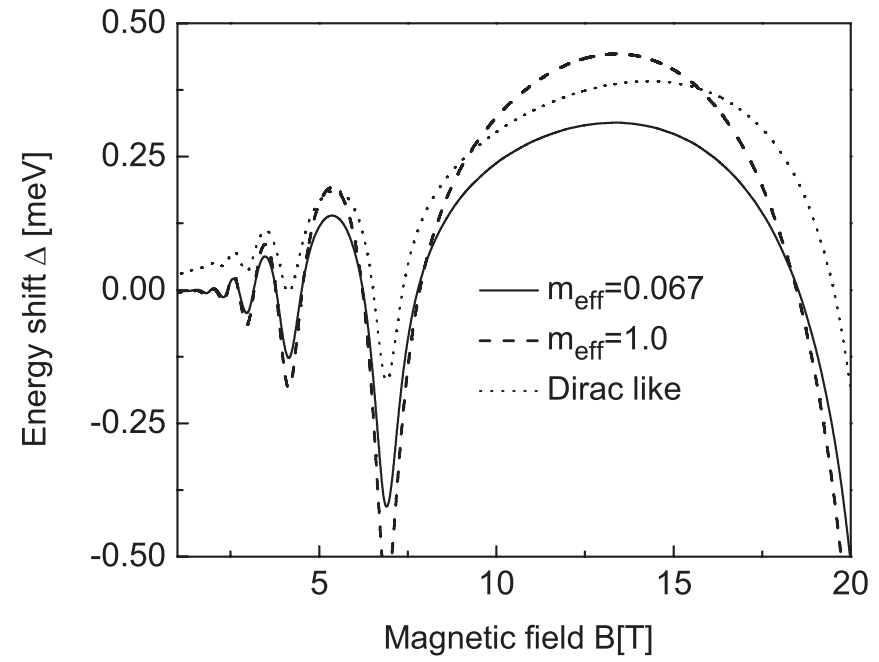

FIG. 6. The energy shift $\Delta$ due to the magnetic field as a function of the magnetic field $B$ for three cases: (1) the remote electron has the mass in GaAs (solid curve), (2) the remote electron behaves like a free electron (dashed curve), and (3) the remote electron with a Dirac-like energy dispersion (dotted curve). Parameters are $n_{s}=10^{12} \mathrm{~cm}^{-2}$ and $A=100$.

Next we investigate the effect of the dispersion relation of the remote electron by considering cases in which the remote electron has the mass of a free electron, or a Dirac-like energy dispersion. The latter could be important for tunneling or interaction between two layers of graphene and can also been seen in the results of the previous figure, Fig. 5. Note that in case the electron does not have a parabolic energy dispersion the formula, Eq. (22) is not applicable and the summation in Eq. (21) has to be performed numerically. In the next figure, Fig. 6, we show the energy shift $\Delta$ in the three following cases: (1) the remote electron has a band mass equal to the GaAs band mass (solid curve), (2) the remote electron has a free electron mass (dashed curve), and (3) the remote electron possesses a Diraclike energy dispersion (dotted curve). In all cases the energy shift exhibits peaks at the same positions, but the difference in absolute value is most pronounced between the two parabolic cases. This is due to the fact that the effective mass $m_{\text {eff }}=$ 0.067 is significantly smaller than that of a free electron.

\section{CONCLUSION}

In this paper we analyzed the interaction between a remote electron and the electrons in single-layer graphene in the presence of a perpendicular magnetic field. The remote electron induces polarization in graphene and the interaction with the layer is described via virtual plasmons. The resulting composite quasiparticle, electron plus polarization, is termed plasmon-polaron. The screening effects in graphene are described by the static dielectric function within the RPA. As a result of this interaction, the ground-state energy of the remote electron is shifted by an amount $\delta E$, which is calculated using second-order perturbation theory. The energy differences, $\Delta$, between the configurations where the electron is placed at $z=d$ and $z=0$, and when $B=0$ are evaluated numerically. Several cases were considered for the remote electron: having the effective mass of GaAs, a free electron mass, and a Dirac-like energy dispersion. In all cases, $\Delta$ oscillates with the magnetic field, which is caused mainly by the dielectric function dependence on the value of $B$. The latter is a consequence of the fact that the screenings are very different for fully and partially occupied Landau levels. The results given in this work are deemed to be useful for further study of electron tunneling into graphene and electron-plasmon interaction. The latter phenomenon attracted a lot of attention recently and is believed to be more pronounced than in conventional semiconductors. It is believed that the present work sheds more light on electron-plasmon interaction in these phenomena.

\section{ACKNOWLEDGMENT}

This work was supported by the Flemish Science Foundation (FWO-Vl).
${ }^{1}$ E. H. Hwang and S. Das Sarma, Phys. Rev. B 80, 205405 (2009).

${ }^{2}$ E. H. Hwang and S. Das Sarma, Phys. Rev. B 75, 205418 (2007).

${ }^{3}$ M. Jablan, M. Soljačić, and H. Buljan, Phys. Rev. B 83, 161409(R) (2011).

${ }^{4}$ M. Polini, R. Asgari, G. Borghi, Y. Barlas, T. Pereg-Barnea, and A. H. MacDonald, Phys. Rev. B 77, 081411 (2008).

${ }^{5}$ A. L. Walter, A. Bostwick, K.-J. Jeon, F. Speck, M. Ostler, T. Seyller, L. Moreschini, Y. J. Chang, M. Polini, R. Asgari, A. H. MacDonald, K. Horn, and E. Rotenberg, Phys. Rev. B 84, 085410 (2011).

${ }^{6}$ K. S. Novoselov, A. K. Geim, S. V. Morozov, D. Jiang, M. I. Katsnelson, I. V. Grigorieva, S. V. Dubonos, and A. A. Firsov, Nature (London) 438, 197 (2005).

${ }^{7}$ Y. Zhang, T.-W. Tan, H. L. Stormer, and P. Kim, Nature (London) 438, 201 (2005)

${ }^{8}$ M. Barbier, F. M. Peeters, P. Vasilopoulos, and J. M. Pereira, Phys. Rev. B 77, 115446 (2008)

${ }^{9}$ M. I. Katsnelson, K. S. Novoselov, and A. K. Geim, Nat. Phys. 2 , 620 (2006).
${ }^{10}$ A. H. C. Neto, F. Guinea, N. M. R. Peres, K. S. Novoselov, and A. K. Geim, Rev. Mod. Phys. 81, 109 (2009).

${ }^{11} \mathrm{Kh}$. Yu Rakhimov, A. Chaves, A. Matulis, G. A. Farias, and F. M. Peeters, J. Phys. Condens. Matter 23, 275801 (2011).

${ }^{12}$ H. Kato, F. M. Peeters, and S. E. Ulloa, Europhys. Lett. 45, 235 (1999).

${ }^{13}$ J. Sak, Phys. Rev. B 6, 3981 (1972).

${ }^{14}$ P. Noziéres and D. Pines, Phys. Rev. 109, 741 (1958).

${ }^{15}$ J. Sabio, J. Nilsson, and A. H. Castro Neto, Phys. Rev. B 78, 075410 (2008).

${ }^{16}$ V. P. Gusynin, S. G. Sharapov, and J. P. Carbotte, Phys. Rev. B 75, 165407 (2007).

${ }^{17}$ C. H. Yang, F. M. Peeters, and W. Xu, Phys. Rev. B 82, 205428 (2010).

${ }^{18}$ N. H. Shon and T. Ando, J. Phys. Soc. Jpn. 67, 2421 (1998).

${ }^{19}$ G. Lindemann, R. Lassnig, W. Seidenbusch, and E. Gornik, Phys. Rev. B 28, 4693 (1983). 